Revue internationale P.M.E.

Économie et gestion de la petite et moyenne entreprise

\title{
Le pilotage de la performance dans les PME en France : une comparaison des pratiques de tableaux de bord des organisations familiales et des filiales
}

\section{Christophe Germain}

Volume 19, numéro 1, 2006

URI : https://id.erudit.org/iderudit/1008490ar

DOI : https://doi.org/10.7202/1008490ar

Aller au sommaire du numéro

Éditeur(s)

Presses de l’Université du Québec

ISSN

0776-5436 (imprimé)

1918-9699 (numérique)

Découvrir la revue

Citer cet article

Germain, C. (2006). Le pilotage de la performance dans les PME en France : une comparaison des pratiques de tableaux de bord des organisations familiales et des filiales. Revue internationale P.M.E., 19(1), 69-94.

https://doi.org/10.7202/1008490ar
Résumé de l'article

À partir des résultats d'une recherche empirique basée sur une enquête quantitative réalisée auprès de 83 entreprises et six études de cas, l'article analyse les caractéristiques des tableaux de bord implantés dans les petites et moyennes entreprises et montre qu'il existe des différences significatives entre les pratiques des entreprises familiales et celles des filiales de groupe. Dans les organisations familiales, les tableaux de bord sont plutôt centralisés, axés sur la performance financière ; ils s'intègrent dans un dispositif de contrôle organisationnel privilégiant l'informel. Dans les filiales, par contre, les tableaux de bord sont plus sophistiqués et plus décentralisés ; ils incorporent des indicateurs non financiers. Cela leur permet d'évaluer la performance sous une forme plus globale et d'être des outils de pilotage au service de la mise en œuvre de la stratégie. 


\title{
Le pilotage de la performance dans les PME en France: une comparaison des pratiques de tableaux de bord des organisations familiales et des filiales
}

Christophe GERMAIN

Audencia Nantes

École de management

\author{
MOTS CLÉS
}

Tableau de bord - Mesure de la performance - Indicateurs

Pilotage - Reporting - PME

\begin{abstract}
RÉSUMÉ
À partir des résultats d'une recherche empirique basée sur une enquête quantitative réalisée auprès de 83 entreprises et six études de cas, l'article analyse les caractéristiques des tableaux de bord implantés dans les petites et moyennes entreprises et montre qu'il existe des différences significatives entre les pratiques des entreprises familiales et celles des filiales de groupe. Dans les organisations familiales, les tableaux de bord sont plutôt centralisés, axés sur la performance financière; ils s'intègrent dans un dispositif de contrôle organisationnel privilégiant l'informel. Dans les filiales, par contre, les tableaux de bord sont plus sophistiqués et plus décentralisés; ils incorporent des indicateurs non financiers. Cela leur permet d'évaluer la performance sous une forme plus globale et d'être des outils de pilotage au service de la mise en œuvre de la stratégie.
\end{abstract}

\section{L'AUTEUR}

Christophe Germain est professeur de contrôle de gestion et directeur adjoint du programme «Grande école» à Audencia Nantes, École de management. II est également membre du CRECCI (Centre de recherches en contrôle et comptabilité internationale) de l'Université Montesquieu Bordeaux IV. Ses recherches portent sur les systèmes de mesure de la performance, notamment des PME et, plus globalement, sur le design du contrôle organisationnel. Adresse: Audencia Nantes, École de Management, 8, route de la Jonelière, B.P. 31222 , 44312 Nantes, Cédex 3, France. Courriel <cgermain@audencia.com>. 


\begin{abstract}
From the results of an empirical research based on a postal survey (83 firms) and six case studies, the paper analyzes the performance measurement systems used in small and medium-sized companies and show that there are differences between the practices of family businesses and subsidiaries. In the entrepreneurial firms, performance measurement systems are often centralized and more used as financial information tools than as strategic management systems, because managers prefer informal control. In subsidiaries, on the other hand, performance measurement systems are more sophisticated and decentralized. They integrate non-financial performance indicators and contribute to implement strategic decision-making.
\end{abstract}

\title{
RESUMEN
}

A partir de los resultados de una investigación empírica basada en una encuesta cuantitativa realizada entre 83 empreses y seis estudios de casos, el artículo analiza las características de los «tableaux de bord" implantados en las pequeñas y medianas empresas y muestra que existe diferencias significativas entre las prácticas de las empresas familiares y las de las firmas que pertenecen a un grupo. En las organizaciones familiares, los «tableaux de bord" estan centralizados, orientados hacia los resultados financieros; se integran en un dispositivo de control organizacional que privilegia lo informal. En las filiales, al contrario, se encuentran "tableaux de bord " más sofisticados y más descentralizados; incluyen indicadores no financieros. Eso les permite evaluar los resultados bajo una forma más global y ser herramientas de pilotaje al servicio de la ejecucion de la estrategia.

\section{ZUSAMMENFASSUNG}

Anhand der Ergebnisse einer empirischen Forschung, die auf einer quantitativen Untersuchung von 83 Unternehmen und sechs Fallstudien basiert, wurden die Eigenschaften der Balanced Scorecard analysiert. Dabei wurden in besonderem Masse die Unterschiede zwischen Familienunternehmen und Filialen aus Unternehmensgruppen betrachtet. Die Untersuchung zeigt, dass erhebliche Unterschiede zwischen den Praktiken der Familienunternehmen und jener der Filialen bestehen. In den Familienorganisationen wird die Balanced Scorecard eher auf die finanzielle Leistung ausgerichtet und ist in eine eher informelle organisatorische Kontrollvorrichtung integriert. In den Filialen dagegen ist die BSC weiter entwickelt und dezentralisiert implementiert. Sie beinhaltet auch nicht finanzielle Indikatoren. Dies erlaubt, die Leistung eines Unternehmens in einer globaleren Form zu evaluieren und erfüllt die Funktion eines Steuerungsinstruments zur Strategieumsetzung.

\section{Introduction}

La problématique de la mesure de la performance a très largement été débattue ces 20 dernières années, au motif qu'il était désormais nécessaire pour les entreprises, compte tenu de l'évolution de l'environnement concurrentiel, de recourir à des mesures non financières de la performance en complément des mesures financières

Revue internationale P.M.E., vol. 19, $\mathrm{n}^{\circ} 1,2006$

(C) 2006 - Presses de l'Université du Québec

Édifice Le Delta I, 2875, boul. Laurier, bureau 450, Québec, Québec G1V 2M2 • Tél.: (418) 657-4399 - www.puq.ca

Tiré de: Revue internationale P.M.E., vol. 19, n 1, sous la direction de Louis Raymond • PME1901N

Tous droits de reproduction, de traduction et d'adaptation réservés 
traditionnelles et de faire évoluer les pratiques de contrôle de gestion. Des systèmes tels que le tableau de bord en France, le balanced scorecard aux États-Unis, le navigateur en Suède ont ainsi été développés ou «redécouverts». De nombreuses recherches ont également été réalisées pour dresser un «état des lieux» des pratiques des entreprises (Kald et Nilsson, 2000; Malmi, 2001; Bescos et Cauvin, 2004), évaluer la diffusion de ces systèmes (Oriot et Misiazeck, 2001; Gehrke et Horvath, 2002; Germain, 2004) ou justifier leur implantation (Ittner et Larcker, 1998; Banker, Potter et Srinivisan, 2000). Ces recherches se sont néanmoins très majoritairement intéressées au cas de la grande entreprise. En revanche, les pratiques de pilotage de la performance des petites et moyennes entreprises demeurent encore aujourd'hui relativement méconnues (Malo, 2000; Reid et Smith, 2000; Chenhall, 2003).

La présente recherche poursuit deux objectifs. Le premier est d'identifier les pratiques de pilotage de la performance des PME en France dans un environnement très imprégné de la culture du «tableau de bord» (Bourguignon, Malleret et Norreklit, 2002). Le second est de comparer les pratiques des structures familiales et des filiales pour évaluer dans quelle mesure et sous quelle forme la propriété du capital peut avoir une influence sur la manière dont la performance est suivie et évaluée dans les petites et moyennes entreprises.

Les résultats obtenus à partir d'une enquête par voie postale réalisée auprès de 89 entreprises et de six études de cas montrent globalement que les tableaux de bord utilisés par les PME sont plutôt décentralisés et réactifs. En revanche, seule une minorité d'entre eux intègre de façon significative des mesures non financières et s'attache à suivre la performance dans sa globalité, comme cela est indiqué dans la littérature. La comparaison des pratiques des organisations familiales et celles des filiales permet de nuancer ces premières conclusions. Il apparaît en effet que les tableaux de bord implantés dans les filiales sont significativement plus développés que les tableaux de bord des structures familiales.

Le tableau de bord est entendu au départ dans une acception très large afin de saisir l'ensemble des formes qu'il peut prendre dans les organisations de taille moyenne. Il est défini comme suit: «un ensemble d'indicateurs choisis et conçus pour permettre à un décisionnaire d'être informé de la performance passée et présente des activités qui entrent dans son champ de responsabilité et des évènements qui peuvent influencer cette performance dans le futur».

L'article comprend quatre sections. Dans la première, nous présentons la problématique de la recherche. Dans la deuxième, nous exposons et justifions la méthode utilisée. Les pratiques des entreprises sont décrites dans la troisième section puis analysées du point de vue des différences mises au jour entre les filiales et les organisations familiales.

Revue internationale P.M.E., vol. 19, $\mathrm{n}^{\circ} 1,2006$

(C) 2006 - Presses de l'Université du Québec

Édifice Le Delta I, 2875, boul. Laurier, bureau 450, Québec, Québec G1V 2M2 • Tél.: (418) 657-4399 - www.puq.ca

Tiré de: Revue internationale P.M.E., vol. 19, no 1, sous la direction de Louis Raymond - PME1901N

Tous droits de reproduction, de traduction et d'adaptation réservés 


\section{La problématique}

Bien que les systèmes de contrôle de gestion traditionnels aient considérablement changé ces dernières années sous l'effet de l'évolution de l'environnement des entreprises, peu de recherches se sont intéressées à la manière dont la performance était suivie et mesurée dans les PME aujourd'hui. Le sujet présente pourtant de l'intérêt puisque les PME évoluent dans un contexte tout aussi complexe et incertain que celui des grandes entreprises. Par ailleurs, il est pertinent pour le traiter de comparer les pratiques des structures familiales et celles des filiales.

\subsection{Systèmes de mesure de la performance et PME}

Le tableau de bord (Lebas, 1994), le balanced scorecard (Kaplan et Norton, 1996a), le navigateur de Skandia (Edvinsson et Malone, 1997) font partie de ce que l'on nomme aujourd'hui les systèmes de mesure de la performance (Bourguignon, Malleret et Norreklit, 2002). Un système de mesure de la performance peut être défini comme une série d'indicateurs financiers et non financiers choisis «pour traduire la mission et la stratégie de l'entreprise en un ensemble de mesures de la performance qui constitue la base d'un système de pilotage de la stratégie» (Kaplan et Norton, 1996a). Ces systèmes ont été développés pour faire évoluer les techniques de contrôle de gestion traditionnelles et satisfaire à une double nécessité née de l'évolution du contexte concurrentiel des entreprises: développer des mesures non financières de la performance et renforcer le lien entre la stratégie et les opérations. Ainsi sont-ils reconnus aujourd'hui comme étant des dispositifs qui développent la réactivité face à des environnements changeants (Chiapello et Delmond, 1994; Rees et Sutcliffe, 1994; Medori et Steeple, 2000), permettent de suivre et d'évaluer la performance dans sa globalité, comme le réclament les stratégies de différenciation (Lorino, 1995; Malina et Selto, 2001), et facilitent la compréhension de la stratégie au niveau des opérationnels (Lorino, 1995; Hall, 2004). Enfin, un certain nombre de travaux indiquent également que l'utilisation de ces systèmes permet d'atteindre une meilleure performance économique (Ittner et Larcker, 1998; Banker, Potter et Srinivisan, 2000; Said, Hassabelnaby et Wier, 2003).

Les systèmes de mesure de la performance ont fait l'objet de nombreuses analyses dans le cadre de la grande entreprise. Les pratiques des PME ont en revanche été peu étudiées (Reid et Smith, 2000; Chenhall, 2003) notamment en France (Malo, 2000), alors que ces entreprises sont confrontées, tout autant que les grandes organisations, à l'incertitude et la complexité de leur environnement, aux nouvelles exigences du marché, à la nécessité d'innover, etc. Levet et Paturel (1997) montrent, par exemple, que pour leur développement, les PME françaises et allemandes privilégient les relations avec les clients et les fournisseurs, la flexibilité, la maîtrise des canaux de distribution, le développement technologique et

Revue internationale P.M.E., vol. 19, nº 1, 2006

(C) 2006 - Presses de l'Université du Québec

Édifice Le Delta I, 2875, boul. Laurier, bureau 450, Québec, Québec G1V 2M2 • Tél.: (418) 657-4399 - www.puq.ca

Tiré de: Revue internationale P.M.E., vol. 19, no 1, sous la direction de Louis Raymond - PME1901N

Tous droits de reproduction, de traduction et d'adaptation réservés 
s'orientent plutôt vers des stratégies où le leadership par les coûts est minoritairement recherché. Schmitt, Julien et Lachance (2002) soulignent par ailleurs que les PME, compte tenu de leurs spécificités, ont la plupart du temps à résoudre des problèmes qui relèvent de situations complexes. Tous ces enjeux composent un contexte où les systèmes de mesure de la performance se révèlent particulièrement adaptés (Chenhall et Morris, 1986; Davila, 2000; Gosselin et Dubé, 2002). Le constat justifie donc que l'on s'intéresse au sujet, sachant qu'il peut être pertinent pour la recherche de comparer les pratiques des entreprises familiales et celles des filiales puisque ces deux types de structures développent des pratiques de gestion différentes.

\subsection{Propriété du capital et pratiques de gestion}

Les résultats d'un certain nombre de travaux indiquent en effet que les entreprises familiales se différencient des autres firmes au plan du comportement stratégique, de la gestion des ressources humaines et du contrôle organisationnel.

Après avoir enquêté auprès d'entreprises familiales et non familiales dans huit pays européens, Donckels et Frölich (1991) constatent, par exemple, que les organisations familiales adoptent un comportement stratégique plus conservateur que les filiales. Le lien entre la forme de propriété et la stratégie est également relevé par Ward (1988) qui montre que les entités familiales adoptent des stratégies de défense pour sauvegarder l'emprise de la famille sur le capital de l'entreprise. En matière de gestion des ressources humaines, Allouche et Amann 1995) observent, quant à eux, des différences significatives entre les organisations familiales et les organisations non familiales. Au sein des premières, il existe une volonté de fidéliser les salariés qui ne se retrouve pas chez les secondes. Cela se traduit par des efforts au plan de la formation et un intérêt pour la valorisation des compétences. Enfin, au plan du contrôle, Goffie et Scase (1985) constatent qu'une des particularités des organisations familiales réside dans le fait que les dirigeants cherchent à renforcer leur contrôle personnel en privilégiant des structures organiques. Cette tendance est également observée par Lyman (1991).

Les enseignements de ces recherches incitent donc à comparer les pratiques de tableaux de bord des entreprises familiales et des filiales pour découvrir les éventuelles différences et les expliquer.

\section{La méthode}

Une enquête a été effectuée auprès d'un échantillon de PME pour recueillir les données nécessaires à la recherche; cette enquête a été complétée par six études de cas. Un instrument a en outre été élaboré pour observer les caractéristiques des tableaux de bord.

Revue internationale P.M.E., vol. 19, nº 1, 2006

(C) 2006 - Presses de l'Université du Québec

Édifice Le Delta I, 2875, boul. Laurier, bureau 450, Québec, Québec G1V 2M2 • Tél.: (418) 657-4399 - www.puq.ca

Tiré de: Revue internationale P.M.E., vol. 19, no 1, sous la direction de Louis Raymond - PME1901N

Tous droits de reproduction, de traduction et d'adaptation réservés 


\subsection{Le terrain}

L'étude empirique a été réalisée auprès d'entités de 10 et 200 salariés, eu égard à la définition de la petite et moyenne entreprise proposée par Marchesnay (1992). Le terrain est composé d'entreprises familiales dont le capital est la propriété du dirigeant (à travers éventuellement un actionnariat familial) et de filiales dont le capital est détenu majoritairement par une autre firme.

\subsection{Le recueil des données}

Une enquête préliminaire a tout d'abord été réalisée sur une période de deux ans auprès de 50 entreprises. Des dirigeants, des contrôleurs de gestion et des responsables comptables ont été interviewés, le but de la démarche étant de définir les attributs des tableaux de bord devant faire l'objet de l'observation et de repérer l'interlocuteur le plus apte à rendre compte des caractéristiques des outils.

Une enquête quantitative a ensuite été effectuée auprès d'un échantillon d'entreprises réparties sur l'ensemble du territoire national français. Ce mode d'accès au terrain a été choisi parce qu'il permettait d'atteindre, en grand nombre, des interlocuteurs (les dirigeants) peu accessibles pour une entrevue (fait constaté lors de l'enquête préliminaire) et d'ainsi recueillir une quantité importante de données. Un questionnaire a été élaboré, puis testé auprès d'un échantillon d'experts en contrôle de gestion et de chefs d'entreprises avant d'être administré par voie postale à 316 dirigeants (en respectant un équilibre entre les deux populations d'entreprises distinguées). Il comporte 50 questions sur l'entreprise elle-même ( $1^{\text {re }}$ partie), ses pratiques en matière de contrôle organisationnel $\left(2^{\mathrm{e}}\right.$ partie $)$, ses pratiques de contrôle de gestion ( $3^{\mathrm{e}}$ partie) et, enfin, ses pratiques de tableaux de bord ( $4^{e}$ partie). Le destinataire de l'enquête a été choisi sur la base des données issues de l'enquête préliminaire, des conclusions de certaines recherches (Chapellier, 1994) et en s'entourant de précautions dans les consignes communiquées au répondant pour compléter le questionnaire ${ }^{1}$. Quatre-vingt-onze questionnaires ont été retournés (taux de réponse de $28,79 \%$ ), dont 83 se sont révélés exploitables. Trente-quatre entreprises familiales et 49 filiales de groupes ont participé à l'enquête.

Six études de cas ont été réalisées ensuite (annexes I et II) pour approfondir les résultats de l'étude quantitative. La combinaison des méthodes de recherche quantitative et qualitative permet en effet de parvenir à une meilleure analyse des phénomènes observés (Allix-Desfautaux, 1998). Dans le cas présent, les études

1. La partie du questionnaire destinée à recueillir des informations sur les caractéristiques instrumentales des tableaux de bord pouvait être complétée par le dirigeant lui-même ou renseignée par un autre responsable, dans le cas où le chef d'entreprise jugeait que sa connaissance des dispositifs en place était insuffisante.

Revue internationale P.M.E., vol. 19, nº 1, 2006

(C) 2006 - Presses de l'Université du Québec

Édifice Le Delta I, 2875, boul. Laurier, bureau 450, Québec, Québec G1V 2M2 • Tél.: (418) 657-4399 - www.puq.ca

Tiré de: Revue internationale P.M.E., vol. 19, no 1, sous la direction de Louis Raymond • PME1901N

Tous droits de reproduction, de traduction et d'adaptation réservés 
de cas ont été effectuées dans le prolongement des résultats de l'enquête, pour analyser le contexte organisationnel et humain dans lequel étaient mis en œuvre les tableaux de bord. Les études de cas concernent trois structures familiales et trois filiales. Les entreprises ont été choisies de façon à pouvoir analyser les pratiques associées aux types de tableaux de bord relevés à l'issue de l'enquête quantitative (Germain, 2003).

\subsection{Les modalités d'observation des tableaux de bord}

Les travaux empiriques antérieurs ne proposant pas d'instruments «clé en main» pour observer les tableaux de bord, un «concept opératoire isolé» (Quivy et Van Campenhoudt, 1995) a été élaboré à partir 1) d'une revue de la littérature consacrée aux systèmes de mesure de la performance, 2) des recherches empiriques sur les systèmes budgétaires et comptables (Chapellier, 1994, 1997) et 3) d'informations recueillies lors de l'enquête préliminaire. Quatre axes d'analyse ont ainsi été choisis pour étudier les tableaux de bord. Ces quatre axes d'analyse sont la réactivité du tableau de bord, la diversité de son contenu, l'étendue de son champ d'analyse (de la performance) et son degré de décentralisation. Ils correspondent aux attributs qui sont habituellement évoqués dans la littérature pour différencier les systèmes de mesure de la performance des systèmes de contrôle de gestion plus traditionnels.

\subsubsection{La réactivité}

Parce qu'il incorpore des données non financières sous la forme d'objectifs à atteindre ou d'indicateurs de suivi de la performance, le tableau de bord fournit de l'information pour agir en amont des résultats financiers (Chiapello et Delmond, 1994). Cette recherche de réactivité est caractéristique des systèmes de mesure de la performance comme le soulignent de nombreux auteurs (White, 1994; Barua, Kriebel et Mukhopadhyay, 1995; Kaplan, 1995; Medori et Steeple, 2000; Said, Hassabelnaby et Wier, 2003). Elle implique que le tableau de bord puisse être diffusé souvent et actualisé rapidement (Chiapello et Delmond, 1994) et que l'outil soit composé d'indicateurs permettant de prévoir les résultats financiers.

La réactivité des tableaux de bord est mesurée dans la recherche en demandant aux répondants d'indiquer quels sont la fréquence et le délai d'édition des tableaux de bord. Cinq modalités de réponse sont proposées pour la fréquence (1. annuelle, 2. trimestrielle, 3. mensuelle, 4 . hebdomadaire, 5 . quotidienne) et le délai (1. un à plusieurs mois, 2. une à deux semaines, 3. plusieurs jours, 4 . un jour, 5. en temps réel). Une évaluation, sur une échelle sémantique différentielle à cinq points allant de 1 (très faible) à 5 (très élevé), du degré de présence des indicateurs prévisionnels et des indicateurs de suivi complète l'appréciation du niveau de réactivité des tableaux de bord. Au final, le degré global de réactivité est évalué en sommant les

Revue internationale P.M.E., vol. 19, $\mathrm{n}^{\circ} 1,2006$

(C) 2006 - Presses de l'Université du Québec

Édifice Le Delta I, 2875, boul. Laurier, bureau 450, Québec, Québec G1V 2M2 • Tél.: (418) 657-4399 - www.puq.ca

Tiré de: Revue internationale P.M.E., vol. 19, n 1, sous la direction de Louis Raymond • PME1901N

Tous droits de reproduction, de traduction et d'adaptation réservés 
scores obtenus sur ces différents items. La valeur de l'alpha de Cronbach obtenue sur cette mesure est de 0,68 ; 1 'instrument peut donc être qualifié de fiable (Usunier, Easterby-Smith et Thorpe, 1993).

\subsubsection{La diversité du contenu}

La nature du contenu des systèmes de mesure de la performance est l'une des caractéristiques qui différencient le plus les systèmes de mesure de la performance des systèmes de contrôle de gestion plus traditionnels (Chiapello et Delmond, 1994; Ittner, Larcker et Randall, 2003). Alors que ces derniers fournissent des données essentiellement comptables et financières, les systèmes de mesure de la performance proposent en complément des données non financières ainsi que des données présentées sous une forme qualitative (clignotants, graphiques, etc.; Chiapello et Delmond, 1994; Mendoza et al., 1999).

La diversité du contenu des tableaux de bord est évaluée ici en demandant aux répondants de décrire au moyen d'une échelle sémantique différentielle à cinq points, le degré de présence dans les tableaux de bord des indicateurs financiers, des indicateurs non financiers et des indicateurs qualitatifs, sachant que chaque type d'indicateurs a été défini très précisément dans le questionnaire. La mesure varie de 1 (très faible) à 5 (très élevé). Au final, la diversité est évaluée en sommant les scores obtenus sur ces trois items. La valeur de l'alpha de Cronbach obtenue sur cette mesure est de 0,68 .

\subsubsection{L'étendue du champ d'analyse}

Grâce à la diversité de leur contenu, les systèmes de mesure de la performance peuvent couvrir plusieurs axes d'analyse de la performance (Malina et Selto, 2001; Ittner, Larcker et Randall, 2003; Hall, 2004). Cette caractéristique est implicite dans le cas du tableau de bord. Elle est en revanche particulièrement saillante dans celui du «balanced scorecard», puisqu'elle structure l'outil en l'orientant a priori vers quatre axes d'analyse: la performance financière, les clients, les processus internes, l'innovation et l'apprentissage (Kaplan et Norton, 1996a).

Pour évaluer la capacité des tableaux de bord à couvrir les composantes de la performance qui ont un lien avec la stratégie de l'entreprise, il a été demandé aux répondants d'indiquer sur une échelle sémantique différentielle à cinq points allant de 1 (très faible) à 5 (très élevé), le degré de présence dans les tableaux de bord des indicateurs relatifs à la performance financière, aux clients, aux processus internes, à l'innovation et à l'apprentissage. Pour les trois derniers types d'indicateurs, le lien avec la stratégie de l'entreprise a systématiquement été évoqué. Dans chaque cas, des exemples ont été proposés pour accroître la clarté des questions et faciliter

Revue internationale P.M.E., vol. 19, nº 1, 2006

(C) 2006 - Presses de l'Université du Québec

Édifice Le Delta I, 2875, boul. Laurier, bureau 450, Québec, Québec G1V 2M2 • Tél.: (418) 657-4399 - www.puq.ca

Tiré de: Revue internationale P.M.E., vol. 19, no 1, sous la direction de Louis Raymond - PME1901N

Tous droits de reproduction, de traduction et d'adaptation réservés 
leur compréhension. L'étendue du champ d'analyse du tableau de bord est évalué ainsi en sommant les scores obtenus sur les quatre items. La valeur de l'alpha de Cronbach obtenue sur cette mesure est de 0,70 .

\subsubsection{Le degré de décentralisation}

Enfin, le degré de décentralisation du tableau de bord est une caractéristique à prendre en compte. Il indique le degré de diffusion de la stratégie dans l'entreprise, sachant qu'il est recommandé d'implanter des systèmes de mesure de la performance aux niveaux hiérarchiques les plus bas de l'entreprise pour que les opérationnels agissent en conformité avec la stratégie de l'entreprise (Chiapello et Delmond, 1994; Kald et Nilsson, 2000; Atkinson, Waterhouse et Wells, 1997).

Le degré de décentralisation des tableaux de bord est évalué en fonction des niveaux hiérarchiques (direction, niveaux fonctionnels, niveaux opérationnels) auxquels sont implantés les outils.

Des données complémentaires ont par ailleurs été recueillies pour évaluer la fréquence et l'intensité d'utilisation des tableaux de bord par les dirigeants.

\section{Les résultats}

Les tableaux de bord des PME sont plutôt réactifs et décentralisés, majoritairement peu diversifiés en contenu et peu étendus au plan du champ d'analyse de la performance. Ils font en outre l'objet d'une utilisation significative de la part des dirigeants qui reconnaissent leur utilité. Ces résultats globaux doivent néanmoins être relativisés puisque les pratiques sont hétérogènes. Il existe en effet des différences notoires entre les tableaux de bord des organisations familiales et ceux des filiales. Les statistiques descriptives des résultats exposés ci-après sont présentées en annexe III.

\subsection{Les caractéristiques instrumentales des tableaux de bord}

\subsubsection{Une recherche de réactivité}

Les tableaux de bord sont actualisés en temps réel dans $27,7 \%$ des entités et dans un délai inférieur à la semaine dans toutes les autres organisations de l'échantillon. Leur fréquence de parution est le plus souvent mensuelle (55,4\% des cas), mais plus d'un tiers des entreprises dispose malgré tout, chaque semaine $(19,3 \%)$ ou chaque jour (19,3\%), de données actualisées. Par ailleurs, la majorité des PME (68,7\%) recourt à des indicateurs de suivi centrés, par exemple, sur le chiffre d'affaires, les marges, la productivité et, dans une moindre mesure, sur les stocks, les coûts et la

Revue internationale P.M.E., vol. 19, n 1, 2006

(C) 2006 - Presses de l'Université du Québec

Édifice Le Delta I, 2875, boul. Laurier, bureau 450, Québec, Québec G1V 2M2 • Tél.: (418) 657-4399 - www.puq.ca

Tiré de: Revue internationale P.M.E., vol. 19, n 1, sous la direction de Louis Raymond • PME1901N

Tous droits de reproduction, de traduction et d'adaptation réservés 
qualité. Enfin, tout en demeurant significatif, le pourcentage des PME disposant d'indicateurs prévisionnels dans des proportions significatives est en revanche plus faible $(48,2 \%)$.

\subsubsection{Une présence significative des indicateurs financiers et, dans une moindre mesure, des indicateurs non financiers}

Les indicateurs financiers demeurent très présents dans les tableaux de bord d'une très grande majorité d'entreprises $(83,1 \%)$. Les indicateurs non financiers sont présents, dans les mêmes proportions, dans 53\% des PME. Ces indicateurs mesurent généralement des variables comme la productivité, les volumes, les délais et la qualité. De ce point de vue, les pratiques des PME ne sont pas si éloignées que cela de celles des grandes entreprises (Bergeron, 1996 ). En revanche, peu de PME (12\%) incorporent des données qualitatives dans les tableaux de bord.

\subsubsection{Un champ d'analyse partiellement global}

Au regard des résultats précédents, il n'est pas surprenant de constater qu'une très grande majorité d'entreprises $(85,5 \%)$ développe des indicateurs pour suivre la performance financière. Une entreprise sur trois $(32,3 \%)$ procède de façon complémentaire à un suivi de la performance des processus internes (qualité, délais, flexibilité, productivité, coûts, etc.). Moins d'un quart des PME (22,9\%) dispose, dans une proportion significative, d'indicateurs relatifs aux clients et au marché. Enfin, très peu d'entités $(4,8 \%)$ concrétisent, par la présence d'indicateurs, la problématique de pilotage de l'innovation et de l'apprentissage organisationnel.

Au regard du cadre d'analyse de la performance défini par Kaplan et Norton (1996a, 1996b), il apparaît donc que les tableaux de bord implantés dans les PME couvrent partiellement les différents champs de la performance. Globalement, il peut être considéré qu'entre un quart et un tiers des PME utilise les tableaux de bord pour la mise en œuvre de la stratégie, comme le recommande la littérature.

\subsubsection{Des dispositifs majoritairement décentralisés}

En matière d'implantation, les tableaux de bord sont présents, dans l'ensemble, à plusieurs niveaux hiérarchiques. Le dirigeant en est le seul destinataire dans très peu de cas $(15,7 \%)$ et les outils sont diffusés aux niveaux des responsables fonctionnels et opérationnels dans respectivement $38,5 \%$ et $45,8 \%$ des entreprises. La décentralisation des dispositifs s'effectue le plus souvent par fonction puis par service et s'accompagne généralement d'une différenciation de contenu (cf. cas 4 et 6 en annexe II).

Revue internationale P.M.E., vol. 19, nº 1, 2006

(C) 2006 - Presses de l'Université du Québec Édifice Le Delta I, 2875, boul. Laurier, bureau 450, Québec, Québec G1V 2M2 • Tél.: (418) 657-4399 - www.puq.ca Tiré de: Revue internationale P.M.E., vol. 19, n 1, sous la direction de Louis Raymond • PME1901N Tous droits de reproduction, de traduction et d'adaptation réservés 


\subsection{L'utilisation des tableaux de bord}

Les tableaux de bord font l'objet d'une utilisation significative de la part des chefs d'entreprise; les fréquences de consultation des outils les plus répandues sont inférieures à la semaine dans $54,2 \%$ des cas. Il semble donc, au regard des conclusions des travaux qui ont analysé les pratiques comptables et de contrôle des PME (Chapellier, 1994 et 1997), que les dirigeants préfèrent les tableaux de bord aux autres instruments de contrôle de gestion lorsqu'ils s'informent de la performance de leur entreprise ${ }^{2}$. Comme cela avait été pressenti, la souplesse et le caractère synthétique des outils expliquent ce résultat qui est confirmé par le fait que la très grande majorité des dirigeants $(64 \%)$ déclare procéder à une analyse du contenu des tableaux de bord dans son entier $(33,8 \%)$ ou pratiquement dans sa totalité $(30,2 \%)$.

Ces premiers résultats doivent cependant être nuancés, car les entreprises familiales possèdent des tableaux de bord moins élaborés que les filiales.

\subsection{Des différences significatives entre les deux groupes d'entreprises}

Les tableaux de bord des filiales sont plus développés que ceux des entreprises familiales. Cela se constate au plan de la réactivité, de la diversité du contenu, du champ d'analyse de la performance et de la décentralisation (voir annexes III et IV).

\subsubsection{Au plan instrumental}

Les tableaux de bord sont en général moins réactifs dans les organisations familiales que dans les filiales. Les délais d'élaboration sont supérieurs à la semaine dans seulement $10,2 \%$ des filiales $(38,3 \%$ des structures familiales); les indicateurs de suivi sont significativement représentés chez la très grande majorité des filiales $(81,6 \%)$, alors qu'une entreprise familiale sur deux les incorpore, dans les mêmes proportions, dans ses tableaux de bord; une majorité de filiales $(55,1 \%)$ dispose en quantités significatives d'indicateurs prévisionnels, contre $38,1 \%$ des organisations familiales.

Si les données financières occupent une place quasi similaire au sein des tableaux de bord des deux types de PME, les données non financières sont plus

2. Chapellier (1994) indique dans sa recherche qu'en règle générale, les dirigeants de PME consultent les données issues des systèmes de coûts à des périodicités qui excèdent le mois et la fréquence d'utilisation des systèmes budgétaires est, quant à elle, beaucoup plus faible.

Revue internationale P.M.E., vol. 19, n 1, 2006

(C) 2006 - Presses de l'Université du Québec

Édifice Le Delta I, 2875, boul. Laurier, bureau 450, Québec, Québec G1V 2M2 • Tél.: (418) 657-4399 - www.puq.ca

Tiré de: Revue internationale P.M.E., vol. 19, $\mathrm{n}^{\circ} 1$, sous la direction de Louis Raymond • PME1901N

Tous droits de reproduction, de traduction et d'adaptation réservés 
représentées dans les outils des filiales que dans ceux des PME familiales; 67,4\% des premières y recourent (contre 32,3\% des secondes). Enfin, les organisations disposant de données qualitatives sont principalement les filiales $(18,4 \%)$.

Cela explique que le champ d'analyse de la performance des tableaux de bord soit en moyenne moins étendu dans les entreprises familiales que dans les filiales. Ces dernières développent plus que les entreprises familiales des indicateurs relatifs aux processus internes $(40,8 \%$ contre $20,6 \%)$ et aux clients $(29,2 \%$ contre $14,7 \%)$. En revanche, les tableaux des deux types d'entreprises possèdent un trait commun: la place réservée aux indicateurs en charge du suivi de l'innovation et de l'apprentissage organisationnel y est très faible.

Enfin, les filiales décentralisent plus leurs tableaux de bord que les entreprises familiales. Ainsi, 55,1\% d'entre elles disposent d'instruments au niveau des responsables opérationnels (contre $23,5 \%$ des structures familiales).

\subsubsection{Au plan de l'utilisation}

Les dirigeants des organisations familiales consultent moins souvent leurs tableaux de bord que leurs homologues des filiales (la fréquence d'utilisation des instruments la plus répandue chez les premiers est le mois $(55,9 \%)$, alors que $36,8 \%$ des seconds déclarent examiner leurs outils tous les jours et $30,6 \%$ toutes les semaines). Par contre, ils procèdent à une analyse plus exhaustive du contenu de leurs outils que les dirigeants des filiales. En effet, 70,6\% d'entre eux affirment prendre connaissance de la totalité des données, alors que la proportion chute à $52,9 \%$ dans le cas des autres chefs d'entreprise. Les entretiens réalisés dans le cadre des études de cas indiquent que les dirigeants des firmes filialisées expriment ici le peu d'intérêt qu'ils accordent aux données de reporting destinées au groupe dont l'utilité est contestée. Comme on l'a déjà constaté dans des travaux antérieurs (Bescos et Mendoza, 1998), c'est moins la surcharge d'information que l'inadaptation de certaines données aux situations de prise de décision qui explique ici l'analyse sélective à laquelle procèdent les chefs d'entreprise.

Les filiales disposent donc de tableaux de bord plus élaborés que les entreprises familiales. Globalement, deux conceptions des outils émergent:

1. Les tableaux de bord des entreprises familiales sont centrés sur la performance financière. Il s'agit d'instruments à vocation informative qui se limitent à l'évaluation ex-post des résultats et ne détiennent pas d'attributions en matière de coordination et d'animation des décisions (liaison stratégie-opérations).

2. Les tableaux de bord des filiales possèdent deux fonctions. Une première catégorie d'indicateurs majoritairement financiers s'intègrent dans les dispositifs de reporting dont la mission principale consiste à informer la hiérarchie et le groupe des résultats de l'entreprise. Les indicateurs de

Revue internationale P.M.E., vol. 19, $\mathrm{n}^{\circ} 1,2006$

(C) 2006 - Presses de l'Université du Québec

Édifice Le Delta I, 2875, boul. Laurier, bureau 450, Québec, Québec G1V 2M2 • Tél.: (418) 657-4399 - www.puq.ca

Tiré de: Revue internationale P.M.E., vol. 19, no 1, sous la direction de Louis Raymond • PME1901N

Tous droits de reproduction, de traduction et d'adaptation réservés 
reporting sont complétés par des indicateurs non financiers qui couvrent plusieurs aspects de la performance et permettent de suivre la mise en œuvre de la stratégie au sein de l'entreprise.

Des publications précédentes (Germain, 2000, 2004, 2005) ont montré que les différences mises au jour ici trouvent leur origine dans les contingences structurelles et comportementales qui s'exercent sur les tableaux de bord. La taille, le secteur d'activité, l'environnement, la stratégie, la structure des entreprises et le style de décision du dirigeant sont des facteurs qui influencent les caractéristiques des tableaux de bord implantés dans les PME. La façon dont les filiales et les organisations familiales définissent, compte tenu de leur système de gouvernement, leurs relations contractuelles avec leurs collaborateurs peut constituer une explication supplémentaire.

\section{L'explication des résultats}

Il est admis que le choix d'une stratégie de contrôle dépend de la manière dont l'entreprise définit et négocie ses relations contractuelles avec ses «partenaires» (clients, fournisseurs, employés, propriétaires, etc.; Atkinson, Waterhouse et Wells, 1997). La mise en place d'un système de mesure de la performance de type «tableau de bord» nécessite ainsi que les règles de collaboration entre les acteurs aient été formulées de façon explicite dans le cadre d'une délégation de responsabilités et qu'il existe, au sein de l'organisation, un référentiel explicite en matière d'interprétation-performance (Lorino, 1995). Le fait que ces conditions soient ou non satisfaites peut constituer, au même titre que le cadre de la théorie de la contingence, une base d'analyse pour expliquer pourquoi les pratiques de tableaux de bord des deux types de PME diffèrent.

\subsection{Les modes de coordination informelle dominent dans l'entreprise familiale}

Dans les entreprises familiales, les tableaux de bord sont peu utilisés pour suivre les actions qui se déroulent en amont des résultats financiers. Cette fonction incombe plutôt à des mécanismes de contrôle informel proches de la supervision directe, pour ce qui est de la coordination verticale, et de l'ajustement mutuel, pour ce qui relève de la coordination horizontale (c'est ce qui a été constaté dans les cas 1, 2, et 3 dans une moindre mesure). La formalisation de la stratégie, des objectifs qui en découlent et des actions à mettre en œuvre, indispensable à la mise en place de tableaux de bord, est moins systématique dans les entreprises familiales que dans les filiales (Kalika, 1987; Calori, Véry et Arrègle, 1997). En l'absence de référentiel formel, il est donc difficile d'implanter des outils de suivi de la performance élaborés. $\mathrm{Ne}$ pouvant pas se fonder sur des paramètres spécifiés, donc mesurables, le contrôle est plutôt réalisé par des mécanismes informels.

Revue internationale P.M.E., vol. 19, nº 1, 2006

(C) 2006 - Presses de l'Université du Québec

Édifice Le Delta I, 2875, boul. Laurier, bureau 450, Québec, Québec G1V 2M2 • Tél.: (418) 657-4399 - www.puq.ca

Tiré de: Revue internationale P.M.E., vol. 19, n 1, sous la direction de Louis Raymond • PME1901N

Tous droits de reproduction, de traduction et d'adaptation réservés 
La situation et l'attitude du dirigeant, dont le rôle est prépondérant dans l'entreprise familiale, peuvent expliquer ce phénomène. Étant plus impliqué dans le capital de l'entreprise que son homologue des filiales, le dirigeant de l'entreprise familiale est plus réticent à recourir à des dispositifs formels pour déléguer son autorité et son pouvoir (Kalika, 1987). Le fait est souligné par Mintzberg (1990, p. 180): «L'organisation individuelle est bien sûr, le cas classique de cette configuration où le leader est aussi le propriétaire. Les entrepreneurs fondent souvent leur propre affaire pour échapper aux procédures et au contrôle de la bureaucratie où ils travaillaient précédemment. À la base de leur propre entreprise, ils continuent à détester les méthodes de la bureaucratie et ses fonctionnels de support logistique ou ses analystes qui vont de pair avec celles-ci et ils maintiennent ainsi leur organisation à une taille modeste et flexible.» Les propos du dirigeant de l'entreprise $n^{\circ} 3$ où un projet d'implantation de tableaux de bord débute illustre ce point de vue: «J'ai créé mon entreprise il y a cinq ans avec 10 salariés au départ. Puis notre activité s'est développée de manière importante et parfois, je le reconnais, anarchique, puisque nos effectifs s'élèvent aujourd'hui à 100 salariés. Mais nos résultats étant très bons, à aucun moment je n'ai ressenti le besoin de me munir d'outils pour analyser de façon détaillée et approfondie notre performance. Aujourd'hui, nous commençons à développer des outils, mais je dois bien avouer que j'ai du mal à me défaire de mon mode de fonctionnement naturel qui me porte à rencontrer les gens et à expliquer plutôt qu'à analyser des tableaux de chiffres ou rédiger des notes.»

En fait, la coordination des activités dans les organisations familiales et les relations entre individus s'établissent plutôt sur la base de représentations informelles communes et d'une confiance réciproque (Allouche et Amann, 1998; Couteret, 1998) qui peut se définir comme «la présomption que, en situation d'incertitude, l'autre partie va agir, y compris face à des situations imprévues, en fonction de règles de comportement acceptables» (Bidault et Jarillo, 1995, p. 113). Cette situation est caractéristique des organisations qui privilégient la «convention» comme mode de régulation, une convention pouvant être définie comme: «une organisation sociale au travers de laquelle la communauté se dote d'une référence commune, produit d'une représentation collective extériorisée qui fonde les anticipations individuelles» (Orléan, 1989, p. 267).

\subsection{Le contexte de la filiale favorise l'implantation de tableaux de bord}

Deux rôles sont attribués aux tableaux de bord dans les filiales. Le premier est lié au reporting, c'est-à-dire à la production périodique d'informations essentiellement financières informant la hiérarchie des résultats de l'entreprise. Le second consiste à suivre des variables qui ont été pointées comme pouvant influer sur le degré d'atteinte des objectifs stratégiques. La volonté d'orienter les actions en fonction

Revue internationale P.M.E., vol. 19, nº 1, 2006

(C) 2006 - Presses de l'Université du Québec

Édifice Le Delta I, 2875, boul. Laurier, bureau 450, Québec, Québec G1V 2M2 • Tél.: (418) 657-4399 - www.puq.ca

Tiré de: Revue internationale P.M.E., vol. 19, no 1, sous la direction de Louis Raymond PME1901N

Tous droits de reproduction, de traduction et d'adaptation réservés 
de la stratégie de l'entreprise, de comprendre et d'anticiper les résultats financiers s'exprime particulièrement dans les propos du dirigeant de l'entreprise $\mathrm{n}^{\circ} 4$ : «Il est très important de lier le financier et le quantitatif. Les données financières ne sont pas forcément parlantes pour un opérationnel. Outre le fait qu'il permet d'expliquer le financier, la quantitatif est le lien avec la stratégie. D'ailleurs, les tableaux de bord que le service de contrôle de gestion édite pour nos différents services sont toujours accompagnés d'une note de synthèse de deux pages qui rappellent les orientations stratégiques de l'année. Ces orientations sont ensuite traduites sous forme d'objectifs dans les tableaux de bord en fonction des indicateurs qui ont été choisis.» En revanche, dans les cas étudiés, il n'existe de dispositifs d'incitations financières associés aux tableaux de bord, comme cela s'observe par exemple aux États-Unis avec le balanced scorecard (Bourguignon, Malleret et Norreklit, 2002).

La mise en place des tableaux de bord s'inscrit, par ailleurs, dans une logique de décentralisation des responsabilités dans la grande majorité des cas (sites $\mathrm{n}^{\text {os }} 4$ et 6 , par exemple). Le contenu et la diffusion des outils varient en fonction des niveaux hiérarchiques et des destinataires afin que chaque responsable dispose, localement et à des périodicités appropriées, de données relatives à son champ d'activité (sites $\mathrm{n}^{\text {os }} 4$ et 6 ). Les informations fournies par les tableaux de bord sont généralement analysées lors des réunions de départements ou de services. Elles sont commentées par les responsables au titre du suivi et de la coordination des actions en cours. Ces informations sont en revanche très rarement utilisées à des fins de retour d'expérience pour engager une réflexion sur les objectifs stratégiques de l'entreprise (Lorino, 1997). Parmi les cas étudiés, seule l'entreprise n ${ }^{\circ} 4$ recourt à cette pratique qui est l'une des formes que peut prendre l'apprentissage organisationnel (Moingeon et Ramanantsoa, 1995).

Globalement, il existe donc dans les filiales une volonté d'utiliser le tableau de bord à des fins managériales et stratégiques. L'analyse du contexte des trois cas étudiés montre que l'implantation des outils est facilitée par le fait que l'organisation a défini et énoncé clairement les modalités de collaboration avec ses responsables aux différents niveaux hiérarchiques de la structure en fonction de la stratégie. Ce préalable est indispensable à la mise en œuvre d'un système de mesure de la performance comme le font remarquer Atkinson, Waterhouse et Wells (1997, p. 80): «La planification aboutit à la négociation de contrats explicites ou implicites avec les parties prenantes. Ces accords stipulent ou sous-entendent à la fois ce que la compagnie attend de chaque groupe d'intéressés pour réaliser ses objectifs primaires et, inversement, ce que chacun attend de l'entreprise en retour de sa collaboration. [...] Les conditions négociées avec chaque groupe ainsi que les apports de chaque processus à la réalisation des objectifs secondaires, déterminent le champ d'application du système de mesure de la performance.» La pression exercée par le groupe joue un rôle déterminant dans la manière dont l'organisation définit ses règles de collaboration avec ses responsables. Devant «rendre des comptes» et produire

Revue internationale P.M.E., vol. 19, $\mathrm{n}^{\circ}$ 1, 2006

(C) 2006 - Presses de l'Université du Québec

Édifice Le Delta I, 2875, boul. Laurier, bureau 450, Québec, Québec G1V 2M2 • Tél.: (418) 657-4399 - www.puq.ca

Tiré de: Revue internationale P.M.E., vol. 19, n 1, sous la direction de Louis Raymond • PME1901N

Tous droits de reproduction, de traduction et d'adaptation réservés 
périodiquement de l'information à destination du groupe, les filiales sont amenées à développer des relations avec leurs collaborateurs qui tendent à être contractuelles, au sens de la théorie de l'agence (Jensen et Meckling, 1976), là où les entreprises familiales s'orientent plutôt vers des mécanismes de coordination plus informels.

\section{Conclusion}

La recherche réalisée auprès d'un échantillon de 83 entreprises françaises de 10 à 200 salariés et à partir de six études de cas montre que les tableaux de bord des PME présentent les caractéristiques suivantes: les outils sont plutôt décentralisés et réactifs, mais seule une minorité d'entre eux intègre de façon significative des mesures non financières permettant de suivre la performance dans sa globalité. Les tableaux de bord possèdent néanmoins des caractéristiques différentes selon qu'ils sont implantés dans des structures familiales ou dans des filiales. Les premières détiennent plutôt des outils à caractère financier et comptable alors que les secondes s'équipent en complément des dispositifs de reporting, de véritables systèmes de pilotage de la performance.

La principale limite de la recherche tient au caractère déclaratif des données collectées; il se peut qu'il existe un écart entre les informations recueillies et la réalité des pratiques. Néanmoins, des mesures ont été prises pour minimiser ce risque et le phénomène n'a pas été constaté lors de l'enquête préliminaire ni lors de la réalisation des études de cas.

La recherche permet donc de cerner les pratiques de tableaux de bord des PME françaises. Au regard des résultats et de l'engouement actuel suscité par les systèmes de mesure de la performance, il est tentant de conclure à un souséquipement des structures familiales et de suggérer à ces dernières de se doter d'outils plus sophistiqués. Il convient malgré tout de rester prudent, car il est admis, au moins depuis les travaux de Lawrence et Lorsch (1973), qu'il n'existe pas de «voie universelle» tant au plan organisationnel qu'en matière de contrôle. La variété doit primer l'uniformité et la qualité du contrôle se construit vraisemblablement plus sur la complémentarité des composantes du contrôle organisationnel et leur congruence avec leur environnement qu'elle ne se décrète à travers la mise en place systématique d'outils élaborés. La gestion de l'organisation du contrôle est en effet aussi importante que le contrôle de la gestion de l'organisation (Burlaud, 2000).

Cette dernière remarque amène à proposer une piste de recherche future qui consisterait à analyser dans quelle mesure, sous quelle forme et comment les mécanismes de coordination informelle évoluent lors de la mise en place de projets d'implantation de tableaux de bord ou de transformation de dispositifs existants. Cela permettrait de relever les différents cas de figure qui peuvent se présenter successivement ou simultanément dans le cadre de ce type de démarche et d'adapter le déroulement du projet en conséquence.

Revue internationale P.M.E., vol. 19, nº 1, 2006

(C) 2006 - Presses de l'Université du Québec

Édifice Le Delta I, 2875, boul. Laurier, bureau 450, Québec, Québec G1V 2M2 • Tél.: (418) 657-4399 - www.puq.ca

Tiré de: Revue internationale P.M.E., vol. 19, no 1, sous la direction de Louis Raymond • PME1901N

Tous droits de reproduction, de traduction et d'adaptation réservés 


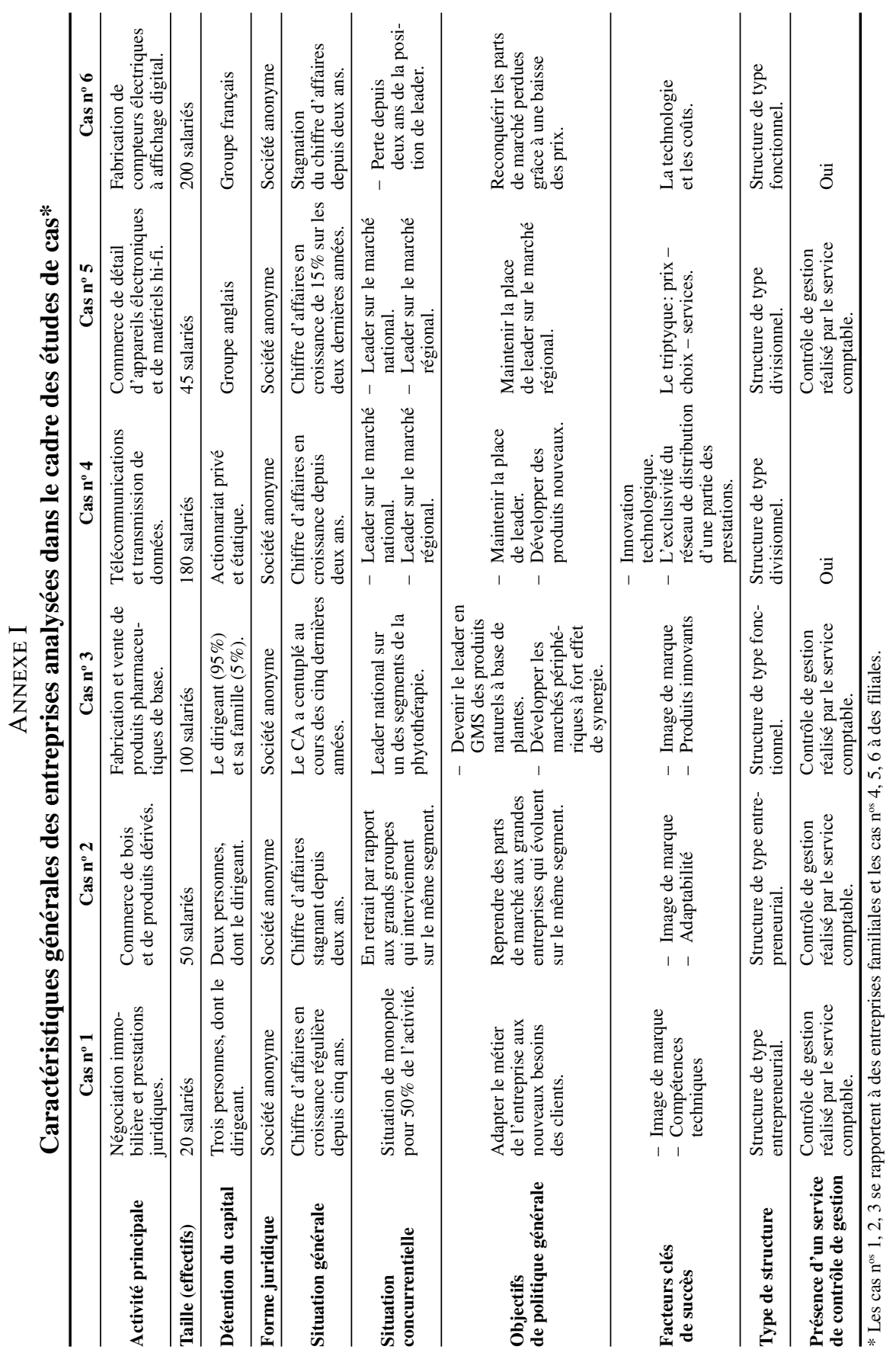

Revue internationale P.M.E., vol. 19, nº 1, 2006

(C) 2006 - Presses de l'Université du Québec

Édifice Le Delta I, 2875, boul. Laurier, bureau 450, Québec, Québec G1V 2M2 • Tél.: (418) 657-4399 - www.puq.ca Tiré de: Revue internationale P.M.E., vol. 19, no 1, sous la direction de Louis Raymond • PME1901N Tous droits de reproduction, de traduction et d'adaptation réservés 


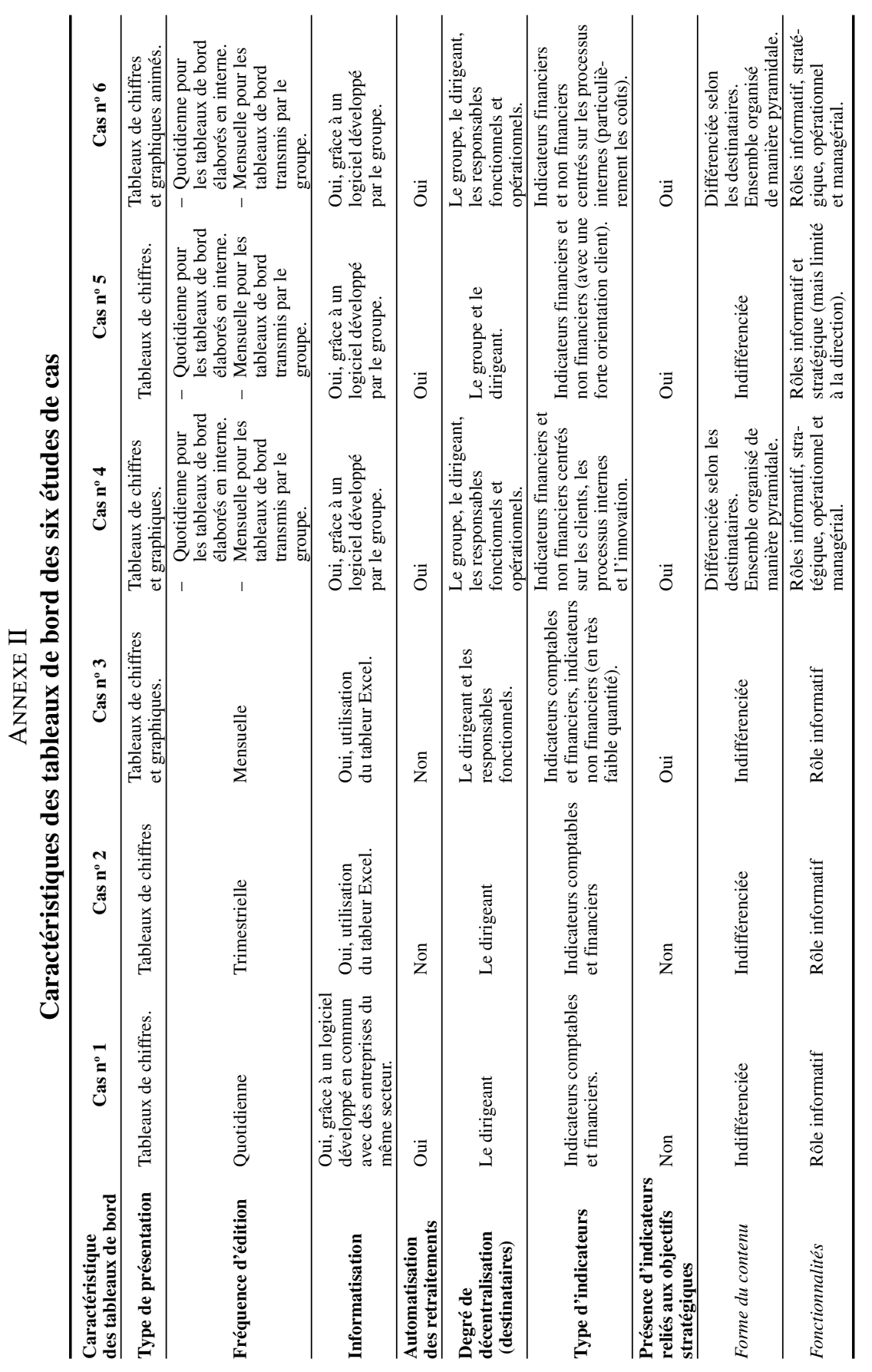

Revue internationale P.M.E., vol. 19, $\mathrm{n}^{\circ}$ 1, 2006

(C) 2006 - Presses de l'Université du Québec

Édifice Le Delta I, 2875, boul. Laurier, bureau 450, Québec, Québec G1V 2M2 • Tél.: (418) 657-4399 - www.puq.ca Tiré de: Revue internationale P.M.E., vol. 19, no 1, sous la direction de Louis Raymond • PME1901N Tous droits de reproduction, de traduction et d'adaptation réservés 
ANNEXE III

Statistiques descriptives des pratiques de tableaux de bord

\begin{tabular}{|c|c|c|c|c|c|c|}
\hline \multirow[t]{2}{*}{ La réactivité } & \multicolumn{2}{|c|}{$\begin{array}{l}\text { Familiales } \\
\qquad(n=34)\end{array}$} & \multicolumn{2}{|c|}{$\begin{array}{c}\text { Filiales } \\
(n=49)\end{array}$} & \multicolumn{2}{|c|}{$\begin{array}{c}\text { Ensemble } \\
(n=83)\end{array}$} \\
\hline & $n$ & $\%$ & $n$ & $\%$ & $n$ & $\%$ \\
\hline \multicolumn{7}{|l|}{ Fréquence de diffusion } \\
\hline - Annuelle & 1 & 2,9 & 1 & 2,1 & 2 & 2,4 \\
\hline - Trimestrielle & 3 & 8,8 & 0 & 0 & 3 & 3,6 \\
\hline - Mensuelle & 21 & 61,8 & 25 & 51 & 46 & 55,4 \\
\hline - Hebdomadaire & 6 & 17,6 & 10 & 20,4 & 16 & 19,3 \\
\hline - Quotidienne & 3 & 8,8 & 13 & 26,5 & 16 & 19,3 \\
\hline \multicolumn{7}{|l|}{ Délai d'élaboration } \\
\hline - Un à plusieurs mois & 4 & 11,8 & 0 & 0 & 4 & 4,8 \\
\hline - Une à trois semaines & 9 & 26,5 & 5 & 10,2 & 14 & 16,9 \\
\hline - Plusieurs jours & 8 & 23,5 & 20 & 40,8 & 28 & 33,7 \\
\hline - Un jour & 7 & 20,6 & 7 & 14,3 & 14 & 16,9 \\
\hline - En temps réel & 6 & 17,6 & 17 & 34,7 & 23 & 27,7 \\
\hline \multicolumn{7}{|c|}{ Présence des indicateurs de suivi } \\
\hline - Très faible & 3 & 8,8 & 2 & 4,1 & 5 & 6 \\
\hline - Faible & 4 & 11,8 & 2 & 4,1 & 6 & 7,2 \\
\hline - Moyenne & 10 & 29,4 & 5 & 10,2 & 15 & 18,1 \\
\hline - Élevée & 8 & 23,5 & 19 & 38,8 & 27 & 32,5 \\
\hline - Très élevée & 9 & 26,5 & 21 & 42,8 & 30 & 36,2 \\
\hline \multicolumn{7}{|c|}{ Présence des indicateurs prévisionnels } \\
\hline - Très faible & 7 & 20,6 & 5 & 10,2 & 12 & 14,5 \\
\hline - Faible & 4 & 11,8 & 5 & 10,2 & 9 & 10,8 \\
\hline - Moyenne & 10 & 29,4 & 12 & 24,5 & 22 & 26,5 \\
\hline - Élevée & 8 & 23,5 & 19 & 38,8 & 27 & 32,5 \\
\hline - Très élevée & 5 & 14,7 & 8 & 16,3 & 13 & 15,7 \\
\hline
\end{tabular}

Revue internationale P.M.E., vol. 19, n 1, 2006

(C) 2006 - Presses de l'Université du Québec

Édifice Le Delta I, 2875, boul. Laurier, bureau 450, Québec, Québec G1V 2M2 • Tél.: (418) 657-4399 - www.puq.ca

Tiré de: Revue internationale P.M.E., vol. 19, $\mathrm{n}^{\circ} 1$, sous la direction de Louis Raymond • PME1901N

Tous droits de reproduction, de traduction et d'adaptation réservés 
ANNEXE III (suite)

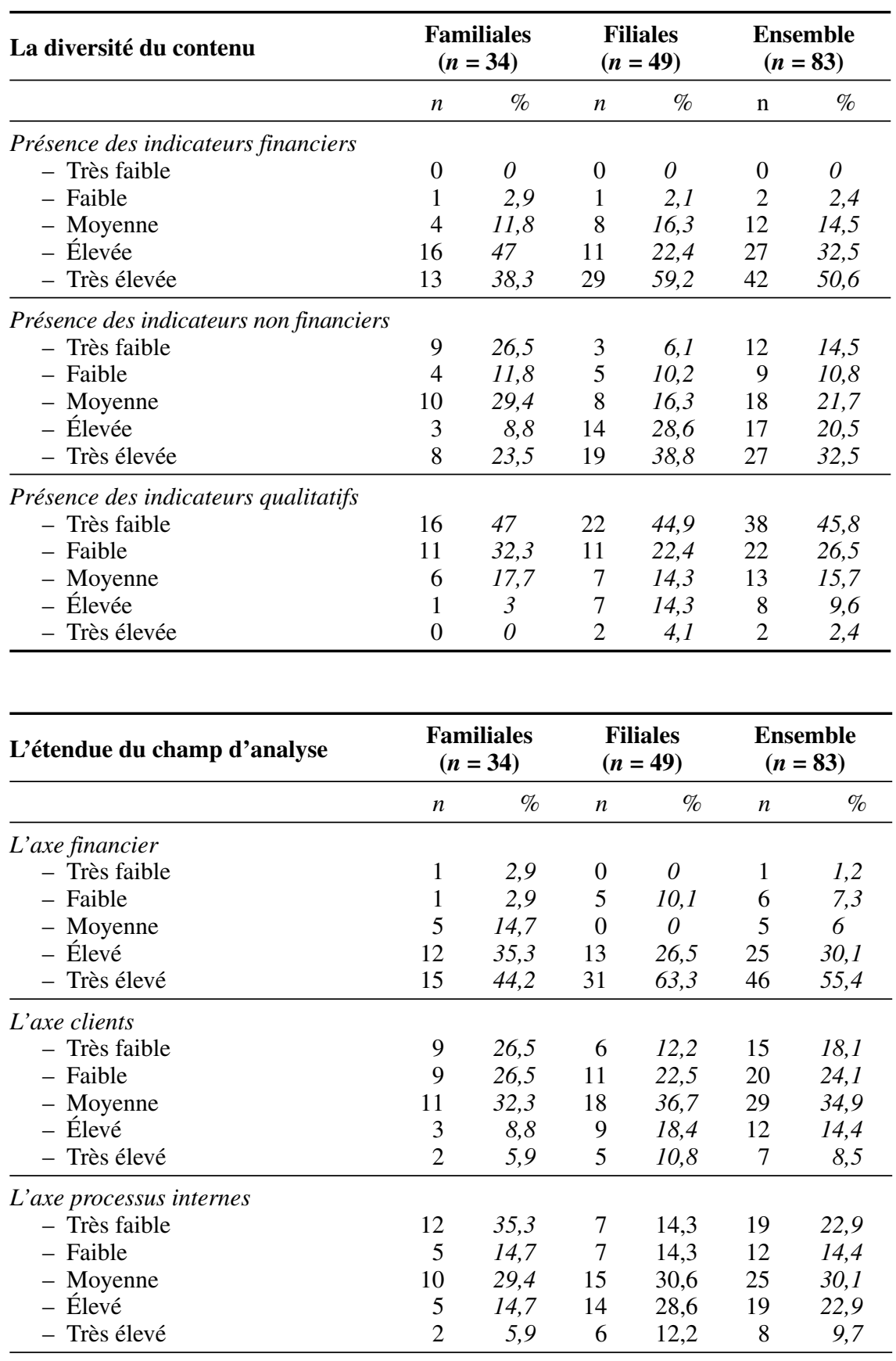


ANNEXE III (suite)

\begin{tabular}{lrrrrrr}
\hline L'axe innovation et apprentissage & & & & & & \\
- Très faible & 15 & 44,1 & 19 & 38,8 & 34 & 41 \\
- Faible & 9 & 26,5 & 16 & 32,6 & 25 & 30,1 \\
- Moyenne & 10 & 29,4 & 10 & 20,4 & 20 & 24,1 \\
- Élevé & 0 & 0 & 3 & 6,1 & 3 & 3,6 \\
- Très élevé & 0 & 0 & 1 & 2,1 & 1 & 1,3 \\
\hline
\end{tabular}

\begin{tabular}{lcrrrrr}
\hline La décentralisation & \multicolumn{2}{c}{$\begin{array}{c}\text { Familiales } \\
(\boldsymbol{n}=\mathbf{3 4})\end{array}$} & $\begin{array}{c}\text { Filiales } \\
(\boldsymbol{n}=\mathbf{4 9 )}\end{array}$ & $\begin{array}{c}\text { Ensemble } \\
(\boldsymbol{n}=\mathbf{8 3})\end{array}$ \\
\hline & $n$ & $\%$ & $n$ & $\%$ & $n$ & $\%$ \\
\hline - Direction & 8 & 23,5 & 5 & 10,2 & 13 & 15,7 \\
- Niveaux fonctionnels & 15 & 44,1 & 17 & 34,7 & 32 & 38,5 \\
- Niveaux opérationnels & 11 & 32,4 & 27 & 55,1 & 38 & 45,8 \\
\hline
\end{tabular}

\begin{tabular}{lrrrrrr}
\hline La fréquence d'utilisation & \multicolumn{2}{c}{$\begin{array}{c}\text { Familiales } \\
(\boldsymbol{n}=\mathbf{3 4})\end{array}$} & $\begin{array}{c}\text { Filiales } \\
(\boldsymbol{n}=\mathbf{4 9})\end{array}$ & $\begin{array}{c}\text { Ensemble } \\
(\boldsymbol{n}=\mathbf{8 3})\end{array}$ \\
\hline & $n$ & $\%$ & $n$ & $\%$ & $n$ & $\%$ \\
\hline - Jamais & 0 & 0 & 0 & 0 & 0 & 0 \\
- Une fois par année & 1 & 2,9 & 0 & 0 & 1 & 1,2 \\
- Une fois par trimestre & 2 & 5,9 & 1 & 2 & 3 & 3,6 \\
- Chaque mois & 19 & 55,9 & 15 & 30,6 & 34 & 41 \\
- Chaque semaine & 9 & 26,5 & 15 & 30,6 & 24 & 28,9 \\
- Chaque jour & 3 & 8,9 & 18 & 36,8 & 21 & 25,3 \\
\hline
\end{tabular}

\begin{tabular}{lrrrrrr}
\hline L'intensité d'utilisation & \multicolumn{2}{c}{$\begin{array}{c}\text { Familiales } \\
(\boldsymbol{n}=\mathbf{3 4})\end{array}$} & $\begin{array}{c}\text { Filiales } \\
(\boldsymbol{n}=\mathbf{4 9 )}\end{array}$ & $\begin{array}{c}\text { Ensemble } \\
(\boldsymbol{n}=\mathbf{8 3})\end{array}$ \\
\hline & $n$ & $\%$ & $n$ & $\%$ & $n$ & $\%$ \\
\hline - Très faible & 0 & 0 & 6 & 12,2 & 6 & 7,2 \\
- Faible & 2 & 5,9 & 3 & 6,1 & 5 & 6 \\
- Moyenne & 8 & 23,5 & 11 & 22,5 & 19 & 22,9 \\
- Élevée & 10 & 29,4 & 15 & 30,6 & 25 & 30,1 \\
- Très élevée & 14 & 41,2 & 14 & 28,6 & 28 & 33,8 \\
\hline
\end{tabular}

Revue internationale P.M.E., vol. 19, $\mathrm{n}^{\circ}$ 1, 2006

(C) 2006 - Presses de l'Université du Québec

Édifice Le Delta I, 2875, boul. Laurier, bureau 450, Québec, Québec G1V 2M2 • Tél.: (418) 657-4399 - www.puq.ca

Tiré de: Revue internationale P.M.E., vol. 19, $\mathrm{n}^{\circ} 1$, sous la direction de Louis Raymond • PME1901N

Tous droits de reproduction, de traduction et d'adaptation réservés 


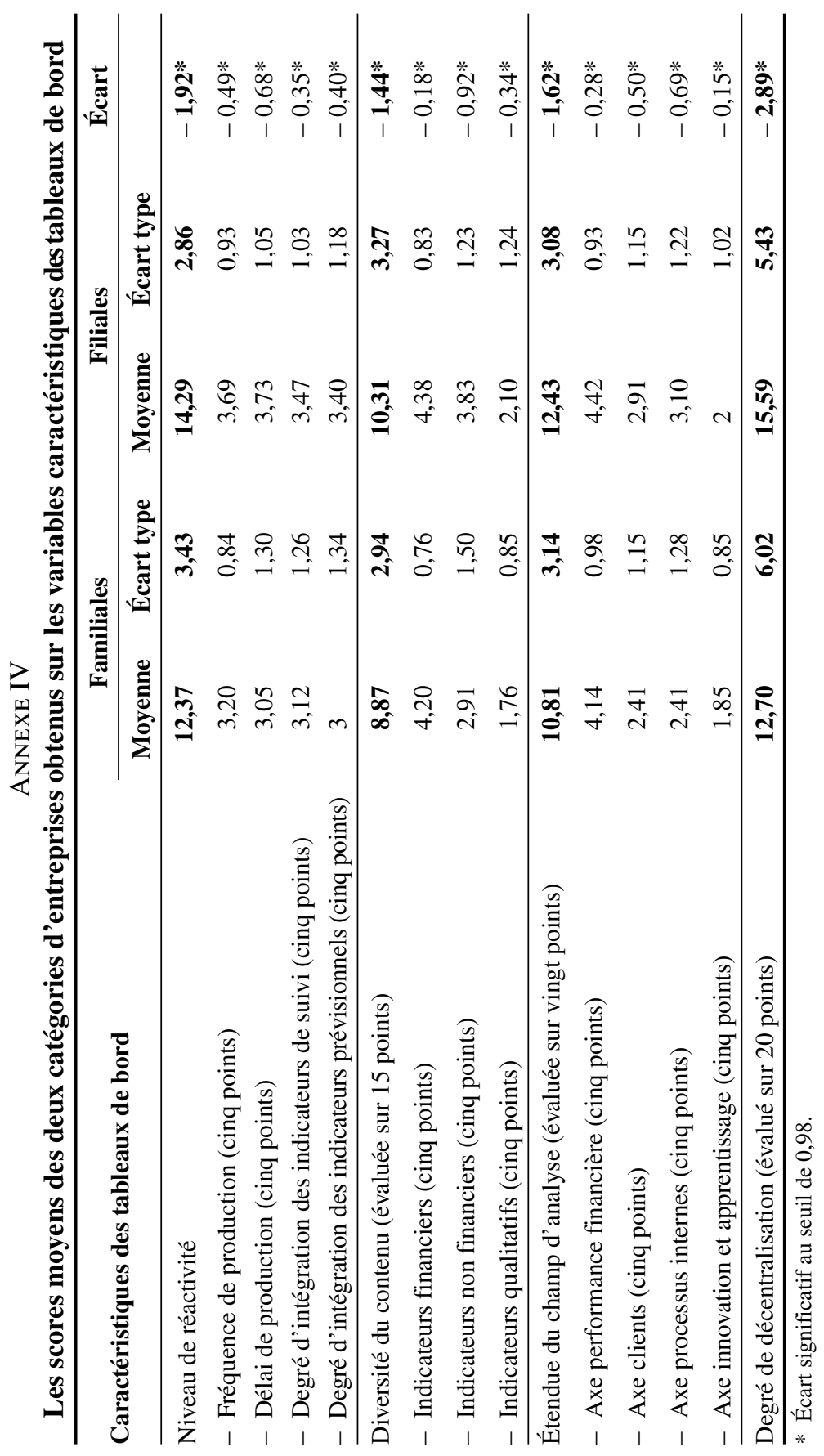

Revue internationale P.M.E., vol. 19, nº 1, 2006

(C) 2006 - Presses de l'Université du Québec

Édifice Le Delta I, 2875, boul. Laurier, bureau 450, Québec, Québec G1V 2M2 • Tél.: (418) 657-4399 - www.puq.ca

Tiré de: Revue internationale P.M.E., vol. 19, $\mathrm{n}^{\circ}$ 1, sous la direction de Louis Raymond • PME1901N Tous droits de reproduction, de traduction et d'adaptation réservés 


\section{Bibliographie}

Allix-Desfautaux, C. (1998), «Triangulation: vers un dépassement de l'opposition qualitatif/quantitatif», Économies et Sociétés, Sciences de gestion, Série S.G., nº 2, p. 209-226.

Allouche, J. et B. AmanN (1995), «Le retour triomphant du capitalisme familial», dans de Jacques Cour à Renault: gestionnaires et organisations, Toulouse, Presses de l'Université des Sciences sociales de Toulouse I.

Allouche, J. et B. Amann (1998), «La confiance, une explication aux performances des entreprises familiales », Économies et Sociétés, Sciences de gestion, Série S.G., ${ }^{\text {os }} 8-9$, p. 129-154.

Atkinson, A., J. Waterhouse et R. Wells (1997), «Bâtir les nouveaux indicateurs de la performance globale», L'Expansion Management Review, n 87, décembre, p. 78-87.

Banker, R.D., G. Potter et D. SRinivisan (2000), «An empirical investigation of an incentive plan that includes nonfinancial performance measures», The Accounting Review, vol. 75, $\mathrm{n}^{\circ}$ 1, p. 65-92.

Barua, A., C.H. Kriebel et T. Mukhopadhyay (1995), «Information technologies and business value: an analytic and empirical investigation», Information Systems Research, vol. 6, $\mathrm{n}^{\circ}$ 1, p. 3-24.

BERGERON, H. (1996), Différenciation des systèmes de données et représentations en contrôle de gestion, Thèse de doctorat, Université de Montpellier II.

Bescos, P.L. et E. Cauvin (2004), «Performance measurement in French companies: an empirical study», Communication présentée au XXVII ${ }^{\mathrm{e}}$ Congrès de l'European Accounting Association, Prague, du $1^{\text {er }}$ au 3 avril.

Bescos, P.L. et C. Mendoza (1998), «Les besoins d'informations des managers sont-ils satisfaits?», Revue française de gestion, $\mathrm{n}^{\circ}$ 121, novembre-décembre, p. 117-128.

Bidault, F. et C. JARILlo (1995), «La confiance dans les transactions économiques», dans Confiance, entreprise et société, Paris, Éditions Eska.

Bourguignon, A., V. Malleret et H. Norreklit (2002), «L'irréductible dimension culturelle des instruments de gestion: l'exemple du tableau de bord et du balanced scorecard», Comptabilité, Contrôle, Audit, numéro spécial, mai, p. 7-37.

Burlaud, A. (2000), «Contrôle et gestion», dans B. Colasse, Encyclopédie de comptabilité, contrôle de gestion et audit, Paris, Economica, p. 521-532.

CAlori, R., P. VÉry et J.L. Arrègle (1997), «Les PMI face à la planification stratégique», Revue française de gestion, $\mathrm{n}^{\circ} 112$, janvier-février, p. 11-23.

Chapellier, P. (1994), Comptabilité et système d'information du dirigeant de PME: essai d'observation et d'interprétation des pratiques, Thèse de doctorat en sciences de gestion, Université de Montpellier II.

Chapellier, P. (1997), «Profils de dirigeants et données comptables de gestion en PME», Revue internationale PME, vol. 10, $\mathrm{n}^{\circ}$ 1, p. 9-41.

Revue internationale P.M.E., vol. 19, nº 1, 2006

(C) 2006 - Presses de l'Université du Québec

Édifice Le Delta I, 2875, boul. Laurier, bureau 450, Québec, Québec G1V 2M2 • Tél.: (418) 657-4399 - www.puq.ca

Tiré de: Revue internationale P.M.E., vol. 19, no 1, sous la direction de Louis Raymond • PME1901N

Tous droits de reproduction, de traduction et d'adaptation réservés 
Chenhall, R.H. (2003), «Management control systems design within its organizational context: findings from contingency-based research and directions for the future», Accounting, Organizations and Society, vol. 28, $\mathrm{n}^{\text {os }} 2-3$, p. 127-168.

Chenhall, R.H. et D. Morris (1986), «The impact of structure, environment and interdependence on the perceived usefulness of management accounting systems », The Accounting Review, vol. 66, $\mathrm{n}^{\circ}$ 1, p. 16-35.

Chiapello, E. et M.H. Delmond (1994), «Les tableaux de bord de gestion, outils d'introduction du changement», Revue française de gestion, janvier-février, $\mathrm{n}^{\circ}$ 97, p. 49-58.

Couteret, M. (1998), «Gérer les ressources humaines de la petite entreprise par la confiance», Revue internationale PME, vol. $11, \mathrm{n}^{\text {os }} 2-3$, p. 95-111.

DAvila, T. (2000), «An empirical study on the drivers of management control system's design in new product development», Accounting, Organization and Society, vol. 25, $\mathrm{n}^{\text {os }} 4-5$, p. $383-409$.

DonCKels, R. et E. FröHLICH (1991), «Are family business really different? European experiences from stratos», Family Business Review, vol. 4, n 2, p. 149-160.

Edvinsson, L. et M.J. Malone (1997), Intellectual Capital: Realizing Your Company's True Value by Finding Its Hidden Brainpower, New York, HarperBusiness.

GehrKe, I. et P. Horvath (2002), «Implementation of performance measurement: a comparative study of French and German organizations», dans M.J. Epstein et J.F. Manzoni, Studies in Managerial and Financial Accounting, vol. 12, p. 159-180, Oxford, JAI Press/Elsevier.

Germain, C. (2000), Contrôle organisationnel et contrôle de gestion: la place des tableaux de bord dans le système de contrôle des petites et moyennes entreprises, Thèse de doctorat en sciences de gestion, Université de Montesquieu Bordeaux IV.

Germain, C. (2003), «Performance measurement and enterprise models: example of dashboards», Communication présentée au XXVI ${ }^{\mathrm{e}}$ Congrès de l'European Accounting Association, Séville, du $1^{\text {er }}$ au 4 avril.

Germain, C. (2004), «La contingence des systèmes de mesure de la performance: les résultats d'une recherche empirique sur le secteur des PME», Finance, contrôle, stratégie, vol. 7, $\mathrm{n}^{\circ}$ 1, mars, p. 33-52.

Germain, C. (2005), «The impact of size, environment, structure and technology on performance measurement systems (PMSs) design: an empirical study of French small and medium-sized enterprises (SMEs)», Communication présentée au XXVIII ${ }^{\mathrm{e}}$ Congrès de l'European Accounting Association, Göteborg, 18-21 mai.

Goffie, R. et R. ScASE (1985), «Proprietorial control in family firms: some fonctions of "quasi-organic" management system», Journal of Management Studies, vol. 22, nº 1, p. 53-68.

Gosselin, M. et T. DubÉ (2002), «Influence de la stratégie sur l'adoption des mesures de performance en vigueur dans le système de comptabilité de gestion», Communication présentée au XXIII ${ }^{\mathrm{e}}$ Congrès de l'Association française de comptabilité, Toulouse, 16 et 17 mai.

Revue internationale P.M.E., vol. 19, nº 1, 2006

(C) 2006 - Presses de l'Université du Québec

Édifice Le Delta I, 2875, boul. Laurier, bureau 450, Québec, Québec G1V 2M2 - Tél.: (418) 657-4399 - www.puq.ca

Tiré de: Revue internationale P.M.E., vol. 19, n 1, sous la direction de Louis Raymond • PME1901N

Tous droits de reproduction, de traduction et d'adaptation réservés 
HALl, M. (2004), «An empirical investigation of the relationship between strategic performance measurement systems, role clarity, psychological empowerment and work outcomes», Document de travail, Université de Melbourne.

ITTNER, C.D. et D.F. LARCKER (1998), «Are nonfinancial measures leading indicators of financial performance? An analysis of customer satisfaction », Journal of Accounting Research, vol. 36, $\mathrm{n}^{\circ}$ 3, p. 1-35.

ITTNER, C.D., D.F. LARCKER et T. RANDALL (2003), «Performance implications of strategic performance measurement in financial services firms», Accounting, Organizations and Society, vol. 28, $\mathrm{n}^{\text {os }}$ 7-8, p. 715-741.

JENSEN, M.C. et W. MeCKLING (1976), «Theory of the firm, managerial behavior, agency costs and ownership structure», Journal of Financial Economics, vol. 3, n 4, p. 305-360.

KALD, M. et F. NiLSSON (2000), «Performance measurement at Nordic companies», European Management Journal, vol. 18, nº 1, p. 113-127.

Kalika, M. (1987), Structures d'entreprises, réalités, déterminants, performances, Paris, Economica.

KAPLAN, R.S. (1995), «Des contrôleurs de gestion aux managers de coûts», L'Expansion Management Review, no 79, décembre, p. 46-52.

Kaplan, R.S. et D.P. Norton (1996a), The Balanced Scorecard, Boston, The Harvard Business School Press.

KAPLAN, R.S. et D.P. NorTON (1996b), «Using the balanced scorecard as a strategic management system», Harvard Business Review, vol. 74, $\mathrm{n}^{\circ}$ 1, janvier-février, p. 75-85.

LAWRENCE, P.R. et J.W. LORSCH (1973), Adapter les structures de l'entreprise: intégration ou différenciation, Paris, Les Éditions d'Organisation.

Lebas, M. (1994), «Managerial accounting in France, overview of past tradition and current practice», The European Accounting Review, vol. 3, n 3, p. 471-487.

Levet, J.L. et R. Paturel (1997), «Comparaisons et explications des stratégies des PMI françaises et allemandes», Revue internationale PME, vol. 10, nº 2, p. 81-108.

LORINO, P. (1995), Comptes et récits de la performance: essai sur le pilotage de l'entreprise, Paris, Les Éditions d'Organisation.

LORINO, P. (1997), Méthodes et pratiques de la performance, Paris, Les Éditions d'Organisation.

Lyman, A. (1991), «Customer service: does family ownership make a difference?», Family Business Review, vol. 4, n 3, p. 303-324.

Malina, M.A. et F.H. Selto (2001), «Communicating and controlling strategy: an empirical study of the effectiveness of the balanced scorecard», Journal of Management Accounting Research, vol. 13, p. 47-90.

Malmi, T. (2001), «Balanced scorecard in Finnish companies, some empirical evidence», Management Accounting Research, vol. 12, n 2, p. 207-284.

Malo, J.L. (2000), «Tableaux de bord», dans Encyclopédie de comptabilité, contrôle de gestion et audit, Paris, Economica, p. 1133-1144.

Marchesnay, M. (1992), «La PME: une gestion spécifique?», Problèmes économiques, $\mathrm{n}^{\circ} 2276$, mai, p. 26-32.

Revue internationale P.M.E., vol. 19, nº 1, 2006

(C) 2006 - Presses de l'Université du Québec

Édifice Le Delta I, 2875, boul. Laurier, bureau 450, Québec, Québec G1V 2M2 • Tél.: (418) 657-4399 - www.puq.ca

Tiré de: Revue internationale P.M.E., vol. 19, n 1, sous la direction de Louis Raymond • PME1901N

Tous droits de reproduction, de traduction et d'adaptation réservés 
Marchesnay, M. et C. Fourcade (1997), Gestion de la PME-PMI, Paris, Nathan.

MEDORI, D. et D. STEEPLE (2000), «A framework for auditing and enhancing performance measurement systems», International Journal of Operations \& Production Management, vol. $20, \mathrm{n}^{\circ} 5$, p. 520-533.

Mendoza, C., H. Delmond, F. Giraud et H. Löning (1999), Tableaux de bord pour managers, Paris, Groupe Revue fiduciaire.

MintzBeRG, H. (1990), Le management: voyage au centre des organisations, Paris, Les Éditions d'Organisation.

Moingeon, B. et B. Ramanantsoa (1995), «Comment rendre l'entreprise apprenante?», L'Expansion Management Review, $\mathrm{n}^{\circ}$ 78, septembre, p. 96-103.

Oriot, F. et E. Misiazeck (2001), «Mesurer une performance multidimensionnelle: le choix du balanced scorecard chez Matra Marconi Space», Échanges, $\mathrm{n}^{\circ}$ 179, p. 26-29.

Orlean, A. (1989), «Pour une approche cognitive des conventions», Revue économique, vol. 40, $\mathrm{n}^{\circ} 2$, p. 241-272.

Quivy, R. et L. VAN CAMPEnhoudt (1995), Manuel de recherche en sciences sociales, Paris, Dunod.

ReEs, W. et C. Sutcliffe (1994), «Quantitative non-financial information and income measures: the case of long-term contracts », Journal of Business Finance and Accounting, avril, p. 331-347.

Reid, G.C. et J.B. Sмiтн (2000), «The impact of contingencies on managerial accounting systems development», Management Accounting Research, vol. 11, n 4, p. 427-4.

Said, A.A., H.R. Hassabelnaby et B. Wier (2003), «An empirical investigation of the performance consequences of nonfinancial measures», Journal of Management Accounting Research, vol. 15, p. 193-223.

Schmitt, C., P.-A. Julien et R. Lachance (2002), «Pour une lecture des problèmes complexes en PME: approche conceptuelle et expérimentation», Revue internationale $P M E$, vol. 15, no 2, p. 35-62.

Usunier, J.C., M. EASTERBY-SMith et R. ThORPE (1993), Introduction à la recherche en gestion, Paris, Economica.

WARD, J.L. (1988), «The special role of strategic planning for family business», Family Business Review, vol. 1, n 2, p. 105-117.

White, G.P. (1994), «A survey and taxonomy of strategy-related performance measures for manufacturing», International Journal of Operations \& Production Management, vol. $16, \mathrm{n}^{\circ} 3$, p. $42-61$.

Revue internationale P.M.E., vol. 19, nº 1, 2006

(C) 2006 - Presses de l'Université du Québec

Édifice Le Delta I, 2875, boul. Laurier, bureau 450, Québec, Québec G1V 2M2 • Tél.: (418) 657-4399 - www.puq.ca

Tiré de: Revue internationale P.M.E., vol. 19, n 1, sous la direction de Louis Raymond • PME1901N

Tous droits de reproduction, de traduction et d'adaptation réservés 